\title{
Entropy-based model for cognitive systems
}

\author{
Moshe Szweizer and Rivka Schlagbaum \\ CBRE Group Inc., Global Research and Consulting, Auckland, New Zealand \\ (Dated: February 27, 2021) - doi.org/10.31219/osf.io/2tv9j
}

\begin{abstract}
Any cognitive experiment involves two subjects. One is the mind of the person being tested, the other is the information involved in the test. While each influences the other, these two need to be analyzed separately for a full understanding of the cognitive processes to emerge. This is because information on its own displays properties which may be confused with the workings of a mind.

The work presented here looks at information as an independent entity. Without any involvement of the mind, information forms patterns and regularities which manifest themselves during cognitive experiments. Information can arrange itself within a multidimensional space and depending on the number of dimensions exhibit different characteristics. It is shown here that information analysis allows for the reproduction of some elementary cognitive processes like short- and long-term memory, chunking, long term memory categories, the formation of convictions, decision-making processes and mechanisms responsible for the formation of understanding. It is therefore argued here that some cognitive experiments do not relate to the workings of a subject's mind but instead relate to the properties of information itself.
\end{abstract}

\section{Introduction}

A scientist intends to make a measurement. A subject matter is present, and an instrument used. The instrument's needle moves and the scientist assumes that the measurement confirmed the experiment. This is called a preconception, which is an evil all scientists are subjected to. It is extremely difficult to make a measurement and not be predisposed as to the expected result and its interpretation.

In cognitive sciences two major elements are present. The first is a subject, usually a person, being tested. The second is the information that forms a part of the experiment. As it happens, from the studies of a learning child to the observations of an elderly philosopher, cognitive science, deals with the manifestations, processing and storage of information. It also happens that information may be isolated from a subject and treated as an independent entity. This makes a cognitive experiment even more complex.

A cognitive experiment consists of two subjects. One is a person and the other the information, which is processed and stored in that person's mind. In this context, when the needle moves, a cognitive scientist is faced with the question: "Am I measuring an attribute of the subject person, or is this some feature of information that is being measured?" To answer this question, one needs to separate the two subjects and to study each one separately.

Information can be separated from any practical manifestation and studied as an independent entity. In such studies, characteristic properties of information may be established. One of these properties is dimensionality. For example, a collection of "facts" without any other influences can be called "one-dimensional". Many processes contain a collection of factual statements only, and these belong to this group.

It is common for a person to hold "convictions". A set of preexisting views, convictions, believes, all of these constitute another dimension influencing information. Thus, two-dimensional information consists of a collection of "facts" and a collection of "convictions". The presence of convictions introduces bias, prejudice and partiality during a decision-making process.

One can add another dimension by introducing "interpretations". These influence the way information is processed and later stored. In this way, a three-dimensional information representation can be created with "facts", "convictions" and "interpretations" on three separate axes.

Finally, by adding "correlations" a four-dimensional representation is arrived at. Correlations manifest themselves when facts, convictions and interpretations are woven together to form relationships. 
When information is analyzed in the above context, it tends to create patterns. These patterns can be represented graphically which is a help when searching for the understanding of their meaning. For example, a threedimensional representation of information can be drawn as lines and closed loops which show similarity to the way long-term memory organizes itself through categories. The lines provide insight into categories by arranging themselves in patterns, some of which are close to the information source and some further away. The lines far away, particularly if they are independently suspended curves, correspond to "abstract ideas". These abstract ideas are also categories, grouped through similarity. Their common feature is the remoteness of the information source.

The paper looks at information in its pure form and asks, what type of cognitive phenomena can be explained by looking at properties of information independently from a subject person. Thus, information is treated as an independent entity and no reference is made to the workings of a human mind. At the same time, numerous cognitive phenomena are being modelled. It is proposed, therefore, that these may be explained by information self-ordering, rather than via the reference to an activity of a human mind.

While progressing the argument well-known cognitive literature is referenced. This is to indicate observations that were reported as part of cognitive research and interpreted as corresponding to the way a human mind works, which are now explainable in the context of information self-organization. Thus, those well-known and wellestablished assumptions about the workings of a mind can be explained through the studies of the properties of information. The citations are of the older works as these over time, have become entrenched in the cognitive researchers' views, as proven facts that do not need to be re-examined.

To give the above discussion more substance a model is presented in the paper. The model uses a formula taken from Physics and Information Theory and expands its usability through the Chaos Theory. By itself, these are well known in the respective fields and are accepted as holding. It is asked, from a reader who is not familiar with these branches of Physics and Mathematics, to accept these methods at face value. It is not an objective of the paper to produce a new method in mathematics. Rather it is to see if the known methods can be successfully applied to cognitive sciences and to establish what type of cognitive phenomenon can be modelled with these. The following is a short discussion of the underpinning methodology, which may be read at leisure.

Entropy is one of the most fundamental concepts in physics (Caticha, 2015). The notion was introduced in the nineteenth century as a part of the development of thermodynamics. It is the building block of the second law, which over time has been formulated through countless expositions. The law states that any process occurring in nature has a directional flow to it, causing systems to deteriorate with time (Wissner-Gross \& Freer, 2013). To reverse this process an external intervention must take place so to restore the original state.

The second law of thermodynamics is the only law of physics that introduces a directionality of time (Martyushev \& Shaiapin, 2016; Caticha, 2011; Martyushev, 2017). All other laws are time-reversible, meaning the effects of any calculation can, in theory, be reversed and the process can be run in the opposite direction. This leads to the association of the concept of entropy and the requirement of its increase over time with the notion of the arrow of time (Parrondo, 2009; Vilenchik, 2019).

At the microscopic level entropy is associated with probability. This is because entropy represents the amount of order in a system. Highly disorganized and random systems are more probable and have larger entropy. Thus, in physics entropy is closely associated with the degree of order.

The ability to associate entropy with probability allows for the application of the above concepts to other fields of study. For example, the concept of entropy has been successfully applied to information theory by Shannon (Shannon, 1959; Verdu, 1998).

Entropy, being one of the most fundamental concepts, manifests her presence in any form of physical but also theoretical reality (Hartman, Olsen \& Lüscher, 2018). It is, therefore, a strong candidate to be a useful tool when constructing any model in any field of study. Following this inclination, a model based on the Boltzmann-Gibbs entropy formula is proposed in this paper.

In the last half a century, chaos theory became one of the more promising tools developed through mathematical investigation (Feigenbaum, 1978). This is due to its ability to model non-linear processes but also because chaos theory seems to be able to describe real processes much better than the formulations based on the traditional approach through, for example, differential equations (Luque, et al., 2011).

The chaos theory approach is quite standard. It always begins with a formulation of a set of feedback formulae, that is formulae whose results are fed back to themselves through the next iteration. These are iterated with the use of a computer until the sequence either diverges to infinity or attains some values within a finite domain. Thus, in chaos theory, all variables are split into two groupings. Those which provide a convergent sequence. This set is called the basin of convergence, and those which result in a divergent sequence and therefore are outside of this basin. Finally, the values to which the sequence converges is labelled an attractor. An attractor can consist of a single value, a finite collection of numbers or an infinite collection of numbers, in which case it is called chaotic.

The paper presented here takes the Boltzmann-Gibbs entropy formula, expresses it in a feedback form and then applies iterations to find the attractor. This is done firstly for one formula, which is assumed to represent an isolated 
persons' convictions building process. Then, another formula is added and the two are iterated as a pair. This second approach is to represent an interaction between free-will and a set of predefined convictions or opinions held by a person. This leads to a model describing a decision-making process. Next, another formula is added to the mix, this time the contribution of the memory is understood to be represented through such expansion. This allows for the process of learning to be modelled. Finally, a fourth formula is introduced allowing for modelling of the phenomenon of understanding as a process.

The paper is closed with a summary and a discussion of findings.

\section{Materials and Methods}

This chapter is provided as a reference, for the sake of a researcher who wishes to reproduce the results or to follow a similar method and is not needed for the understanding of the general text.

In the case of a two-parameter sequence, for an initial point $\left(\mathrm{p}_{0}, \mathrm{q}_{0}\right)$ the sequence is initiated with (2a) followed by ( $2 b)$ and ( $2 a)$ etc. This makes the " $p$ " sequence never to be exposed to the initial condition of $q_{0}$ as in $q_{1}\left(p_{0}, q_{0}\right)$, $\mathrm{p}_{1}\left(\mathrm{p}_{0}, \mathrm{q}_{1}\right), \mathrm{q}_{2}\left(\mathrm{p}_{1}, \mathrm{q}_{1}\right), \mathrm{p}_{2}\left(\mathrm{p}_{1}, \mathrm{q}_{2}\right) \ldots$ The sequence is iterated 200 times and then, depending on the requirements, either the next 200 iterations or the immediately following value collected. As (2a) is used in the first iteration, the two sequences are asymmetric with the Q-sequence having an advantage of the first move. A similar technique is applied when a higher number of dimensions are modelled.

\section{Single Parameter Entropy Evolution - Facts and their acceptance}

A simple question "What do you know about this?" causes a response in the form of a list of facts. "I know this, this, this and that." A collection of facts constitutes a one-dimensional information space. The facts can be listed as bullet points on a single-dimensional list.

The truthfulness of each fact is accepted to a certain degree. Therefore, there exists a notion of the level of acceptance associated with each fact. The stronger the supporting information, called "witnessing" here, the higher level of acceptability of a factual statement.

Chaos techniques are based on feedback sequences, where the result of a previous calculation is used as an input variable in the subsequent step. In the case of the entropy-based chaos model, this enforces a requirement for entropy to be understood as representing probability as in (1).

$$
q_{n+1} \equiv S=-k q_{n} \ln \left(q_{n}\right)
$$

Here, the standard Boltzmann-Gibbs formula for entropy is used as the basis of the feedback equation and the Boltzmann constant $\mathrm{k}$ is replaced with a parameter constrained within the $(0, \mathrm{e})$ range. In the information theory, when calculating entropy, the RHS would be summed over all possible probability distributions and in this way, the resultant maximum probability would be found. This is achieved here through the iteration, which converges to the most likely outcome which is expressed through the attractor instead.

The limitation on $\mathrm{k}$ is the consequence of the convergence constraint for (1) which diverges for $\mathrm{k}$ larger than the Euler's number. Thus, to recover probability on the LHS of (1) one requires the RHS to be within the $(0,1)$ range. In the model presented here, the k-parameter is understood to relate to the amount of available information. In turn, information depends on time, as it takes time to collect it, and on the strength of supportive witnessing. There could be one, two or more sources of information, labelled here as witnesses, who support or disprove information with various strengths of argument. When the number of witnesses increases above four the confirmation attains a social component.

The attractor achieved through (1) yields the Feigenbaum chart shown in Fig. 1. For this model, $\mathrm{k}$ is assumed to represent the amount of information available and Q the strength of acceptance a person exposed to this information can experience as in Table 1. 


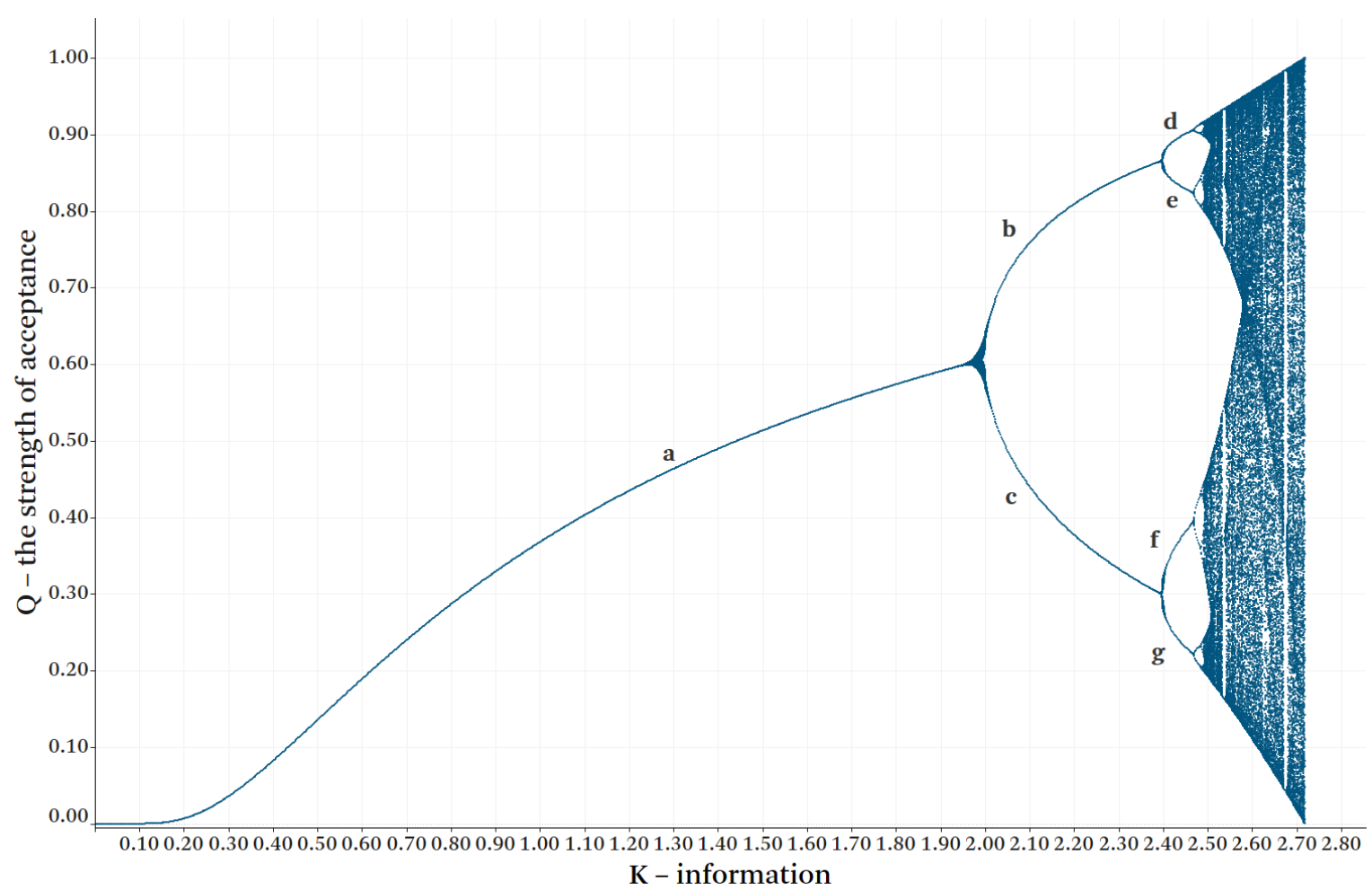

Fig. 1. One parameter Feigenbaum chart

Facts are a part of implicit memory (Atkinson \& Shiffrin, 1968). As such this type of information is held in the memory without conscious awareness, but at the same time, it has a major influence on decision making and interpretation of reality.

Table 1. One parameter attractor components (refer to Fig. 1 for the branch labelling)

\begin{tabular}{|c|c|c|c|}
\hline Branch & $\mathrm{K}$ - range & $\begin{array}{l}\text { Witnessing } \\
\text { (Yes/No) }\end{array}$ & Interpretation \\
\hline $\mathrm{a}$ & $0-1.95$ & $\mathrm{Y}$ & $\begin{array}{l}\text { Acceptance is based on a singular witness whose strength of } \\
\text { argument varies from very weak (0) up to } \mathrm{k}=2 \text {. As this is the only } \\
\text { witness the recipient is never fully convinced or ever fully accepts } \\
\text { it, with the highest value of } 60 \% \text {. }\end{array}$ \\
\hline $\mathrm{b}$ & $1.95-2.39$ & YY & $\begin{array}{l}\text { Another witness supports information bringing the credibility up to } \\
87 \%\end{array}$ \\
\hline $\mathrm{c}$ & $1.95-2.39$ & YN & $\begin{array}{l}\text { The second witness disagrees with the first undercutting the } \\
\text { acceptance. Even though two witnesses are contradicting each other } \\
\text { the subject person would still accept the original claim as possible } \\
\text { even though to a lesser degree. The lowest value is } 30 \% \text {. }\end{array}$ \\
\hline d & $2.40-2.47$ & YYY & $\begin{array}{l}\text { Three witnesses support each other bringing the credibility up to } \\
91 \% \text {. }\end{array}$ \\
\hline $\mathrm{e}$ & $2.40-2.47$ & YYN & $\begin{array}{l}\text { Two witnesses supporting followed by a contradiction. This reduces } \\
\text { acceptance to the } 82 \%-86 \% \text { range. }\end{array}$ \\
\hline f & $2.40-2.47$ & YNY & $\begin{array}{l}\text { Three witnesses, supporting, rejecting and supporting again. Even } \\
\text { though this branch has the same number of supporting and rejecting } \\
\text { witnesses as branch e, the resultant strength of support is different, } \\
\text { illustrating that the order in which the witnesses appear does matter. } \\
\text { Here the acceptance range is } 30 \%-40 \% \text {. }\end{array}$ \\
\hline g & $2.40-2.47$ & YNN & $\begin{array}{l}\text { The first witness is contradicted by the subsequent two. Thus, even } \\
\text { though the number of contradicting witnesses is greater than the } \\
\text { original one the subject person would be still giving some credit to }\end{array}$ \\
\hline
\end{tabular}


the original witnessing and thus keeping it as a possibility.

Acceptance range is $22 \%-30 \%$.

$2.47-2.48 \quad 4(\mathrm{Y} / \mathrm{N}) \quad$ supporting view and somewhat larger to the initially contradicted (c).

Above 4 witnesses a social element takes over and the witnessing is interpreted as a social statement more than an account by

Above 2.48 Social individuals. Thus, when information is supported by many witnesses it may be accepted uncritically at almost $100 \%$ or rejected totally at almost $0 \%$ (if contradicted by all). The chart shows chaos which is initially positioned around (b) and (c) directions.

An important element of Fig. 1 is that there is no gap at k close to zero. That is, some degree of acceptance is present even when the amount of information is negligibly small. This is in contrast with the charts shown in the following sections.

\section{Two Parameter Model - decision making}

The second dimension of information is formed by convictions. These are made of believes and views about reality. While facts constitute abjective statements about truths, convictions are irrational and personal.

When information is expressed in two dimensions with facts plotted against convictions a process of decision making becomes available. During that process, a continuous interaction between facts and convictions is entertained. Facts make a statement, the convictions respond. These are verified against facts and send back to convictions. This interactive process is continued until a decision is arrived at. As convictions are personal, a decision arrived at is also personal.

In the second version of the model, the entropy formula is modified by adding a conditional statement and the interaction between the two components involved is achieved through executing the script as (2a, 2b, 2a, 2b...). This version of the model expands it by adding a parameter $\mathrm{P}$ which allows for the decision-making process to take place. Here, Q corresponds to facts and $\mathrm{P}$ to the set of convictions held by a person. The convictions influence the decision-making process by making it biased towards the opinions held. It can be shown using Lyapunov exponents that the $\mathrm{P}$ component uniformly influences the trajectory in one of the directions, while the Q component experiences clustering at $\mathrm{k}$ close to unity and afterwards moves the line in either direction. This change in direction is interpreted as representing the phase when the decision is elected.

$$
\begin{aligned}
& q_{n+1}=P(q \mid p)=\frac{P(q \cap p)}{P(p)}=\frac{-k q_{n} p_{n} \ln \left(q_{n} p_{n}\right)}{p_{n}}=-k q_{n} \ln \left(q_{n} p_{n}\right) \\
& p_{n+1}=P(p \mid q)=\frac{P(q \cap p)}{P(q)}=\frac{-k q_{n} p_{n} \ln \left(q_{n} p_{n}\right)}{q_{n}}=-k p_{n} \ln \left(q_{n} p_{n}\right)
\end{aligned}
$$

Formulae (2a) and (2b) constitute a two-dimensional feedback set of sequences, which is highly dependent on initial conditions $\mathrm{p}_{0}$ and $\mathrm{q}_{0}$. As the sequence is iterated the values of $\mathrm{p}_{\mathrm{n}}$ and $\mathrm{q}_{\mathrm{n}}$ are constrained through a hyperbole $\mathrm{pq}<=1$ imposed through the natural logarithm. Therefore, $\mathrm{p}$ and $\mathrm{q}$ may attain any large value if their product is bound by the hyperbole. The attractor chart is plotted for the normalized values of p-normalized and q-normalized as in (3a) and (3b).

$$
\begin{aligned}
& q \text { normalised } \stackrel{\text { def }}{=} \frac{q}{q+p} \\
& p \text { normalised } \stackrel{\text { def }}{=} \frac{p}{q+p}
\end{aligned}
$$

\subsection{An example of two-parameter based trajectories}

Fig. 2 presents a selection of q-normalized trajectories for randomly selected initial conditions. The characteristic features of trajectories include not beginning at k equal to exactly zero, as there is a gap of about 0.03 before the attractor commences. At the very beginning, a trajectory contains a scattered distribution of points. 
Any two trajectories which follow a similar path may diverge at a later point and turn rapidly upwards or downwards. At k close to the first bifurcation (Fig. 1, k=2.0) the trajectories diverge to one of the two extremes (0 or 1). This is followed by a period of being constant and then attaining chaos as the last phase.

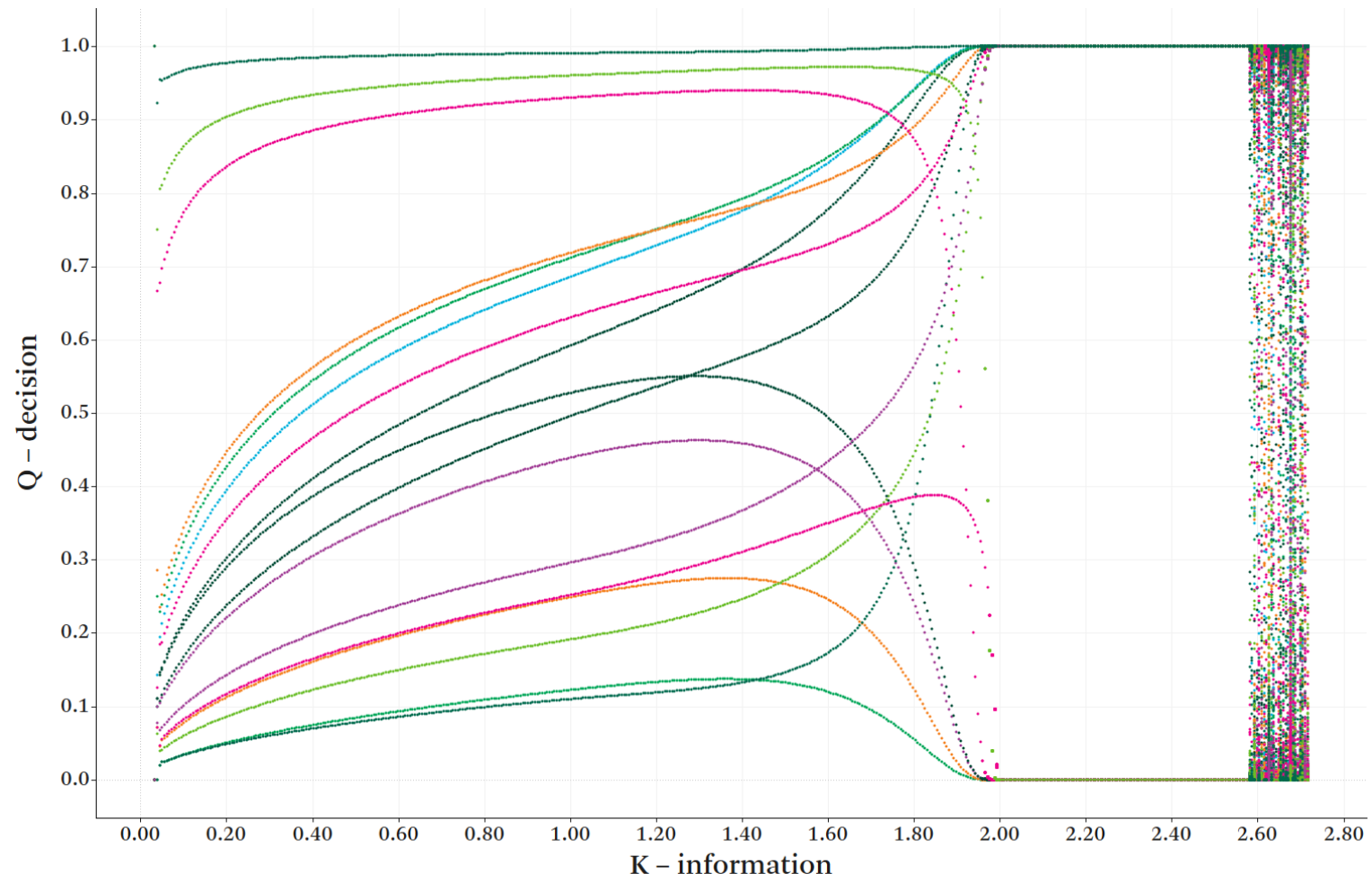

Fig. 2. An example of Q-normalized trajectories

The attractor's behaviour for small $\mathrm{k}$ is shown in Fig. 3. The sequence does not converge for $\mathrm{k}$-values under 0.03. Between 0.03 and 0.04 , the attractor is either zero or unity (not shown but similar). This is followed by a region of scattered points which gather to form a line which is followed as in Fig. 2.

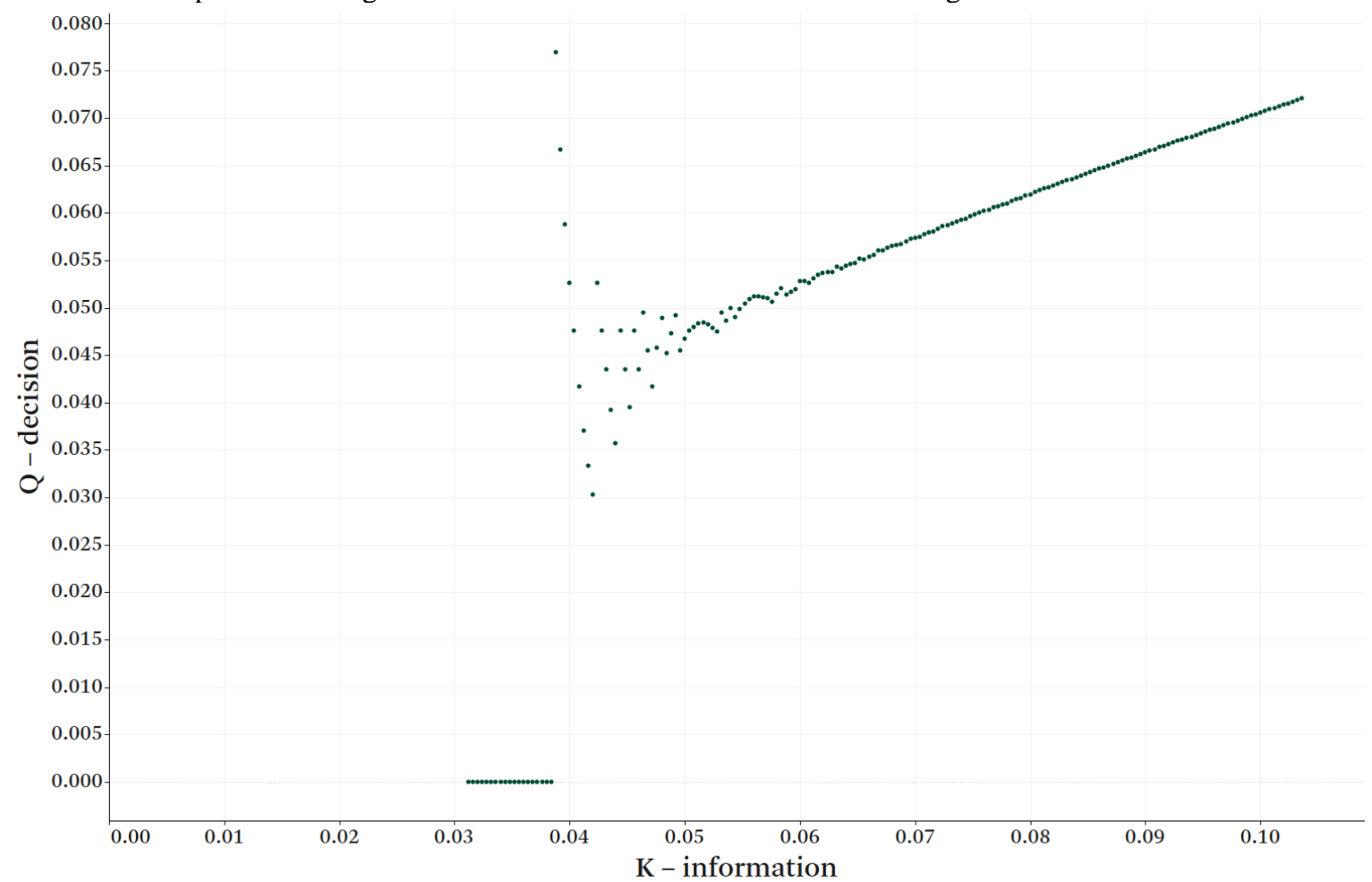

Fig. 3. A sample trajectory at small values of $k$ 
Table 2 summarizes the proposed interpretation of Fig. 2 and Fig. 3.

Table 2. Stages of Decision Making

\begin{tabular}{|c|c|}
\hline $\mathrm{K}$ - information & Interpretation \\
\hline Under 0.03 & $\begin{array}{l}\text { A person needs a non-negligible amount of information even to start considering a } \\
\text { decision. Thus, trajectories do not start at zero, although the amount of information } \\
\text { required for the trajectory to start is very small. }\end{array}$ \\
\hline $0.03-0.04$ & $\begin{array}{l}\text { The model predicts the existence of a kneejerk reaction, which represents a decision } \\
\text { issued as a reflexive response to a limited amount of information. This happens in } \\
\text { reality, even though it may seem irrational and rushed. Fig. } 3 \text { illustrates a rejection } \\
(0-\text { No) but other trajectories include acceptance }(1-\text { Yes }) \text {. }\end{array}$ \\
\hline $0.04-0.07$ & $\begin{array}{l}\text { Classification of the problem. An increase in information narrows down the problem } \\
\text { to a specific trajectory. This creates a scattering of points at the time when the } \\
\text { problem to be addressed is being defined. }\end{array}$ \\
\hline $0.07-1.95$ & $\begin{array}{l}\text { Over this period information is being accumulated so that an educated decision can } \\
\text { be made. At k in the region close to unity a phenomenon which can be described as } \\
\text { "making up one's mind" is observed. At that stage, the Q trajectories cluster together } \\
\text { and subsequently move toward one of the two extreme values (Yes/No). This can be } \\
\text { observed analytically through the analysis of Lyapunov exponents against Q and P } \\
\text { components of the attractor. }\end{array}$ \\
\hline $1.95-2.00$ & $\begin{array}{l}\text { The decision is attained at this stage with the attractor moving closely to }(0,1) \\
\text { values. }\end{array}$ \\
\hline $2.00-2.56$ & $\begin{array}{l}\text { Even though in Fig. } 2 \text { the attractor seems to attain the boundary values, the set is } \\
\text { open, that is points } 0 \text { and } 1 \text { do not belong to the attractor. This is the consequence of } \\
\text { the natural logarithm as in }(2 \mathrm{a}) \text { and ( } 2 \mathrm{~b}) \text { not being defined at zero. For the decision- } \\
\text { making process, this results in the inability of a person to be completely convinced } \\
\text { as to being right or wrong when making any decision. }\end{array}$ \\
\hline $2.56-2.718$ & Chaos sets in - relates to indecision if a person is overwhelmed with information. \\
\hline
\end{tabular}

\section{Three Parameter Model - memory and learning}

The third dimension of information is formed by interpretations. These are assignments of meanings to individual information fragments. The interpretations depend on convictions and known facts, therefore the process can be expressed in three dimensions.

Similarly to the two-dimensional decision-making process, the three-dimensional analysis arrives in steps. Facts are consulted with convictions and the information send to interpretation which in turn sends its findings back to the facts for verification. This cyclic process is continued until all three components find a stable solution. Once information is interpreted it may be stored for future use. Thus memory contains facts and convictions which have been interpreted.

The model is obtained by running the sequence as facts (4a), convictions (4b) and interpretations (4c) in that order. The sequence starts with some initial facts, convictions and interpretation $\left(\mathrm{q}_{0}, \mathrm{p}_{0}, \mathrm{z}_{0}\right)$, and results in a strange attractor as shown in Fig. 4.

$$
\begin{aligned}
& q_{n+1}=P(q \mid z)=\frac{P(q \cap \mathrm{z})}{P(z)}=\frac{-k q_{n} z_{n} \ln \left(q_{n} z_{n}\right)}{z_{n}}=-k q_{n} \ln \left(q_{n} z_{n}\right) \\
& p_{n+1}=P(p \mid q)=\frac{P(q \cap p)}{P(q)}=\frac{-k q_{n} p_{n} \ln \left(q_{n} p_{n}\right)}{q_{n}}=-k p_{n} \ln \left(q_{n} p_{n}\right) \\
& z_{n+1}=P(z \mid p)=\frac{P(\mathrm{z} \cap p)}{P(p)}=\frac{-k z_{n} p_{n} \ln \left(z_{n} p_{n}\right)}{p_{n}}=-k z_{n} \ln \left(z_{n} p_{n}\right)
\end{aligned}
$$

As in (3a) and (3b), the normalization is achieved by dividing by the sum of all variables.

The three-parameter attractor consists of three distinct phases. The first phase occurs at the low end of the krange and relates to the sensory and short-term memory. During this phase, the attractor expresses itself through 
several parallel lines which spin together toward a common central point. These are associated with sensory memory channels which interact with each other during the initial information processing. Depending on the initial conditions, numerous attractor expressions do not progress beyond this phase, therefore it would be natural to associate it with the short-term memory. The number of lines is larger than two, therefore one expects other sensory channels to exist apart of iconic and echoic memory (Sperling, 1960; Cowan, Lichty \& Grove, 1990). Smell and taste, as well as touch, also supply information that is memorized and are expressed through these channels. The short-term memory with evident channels is shown in Fig. 4.

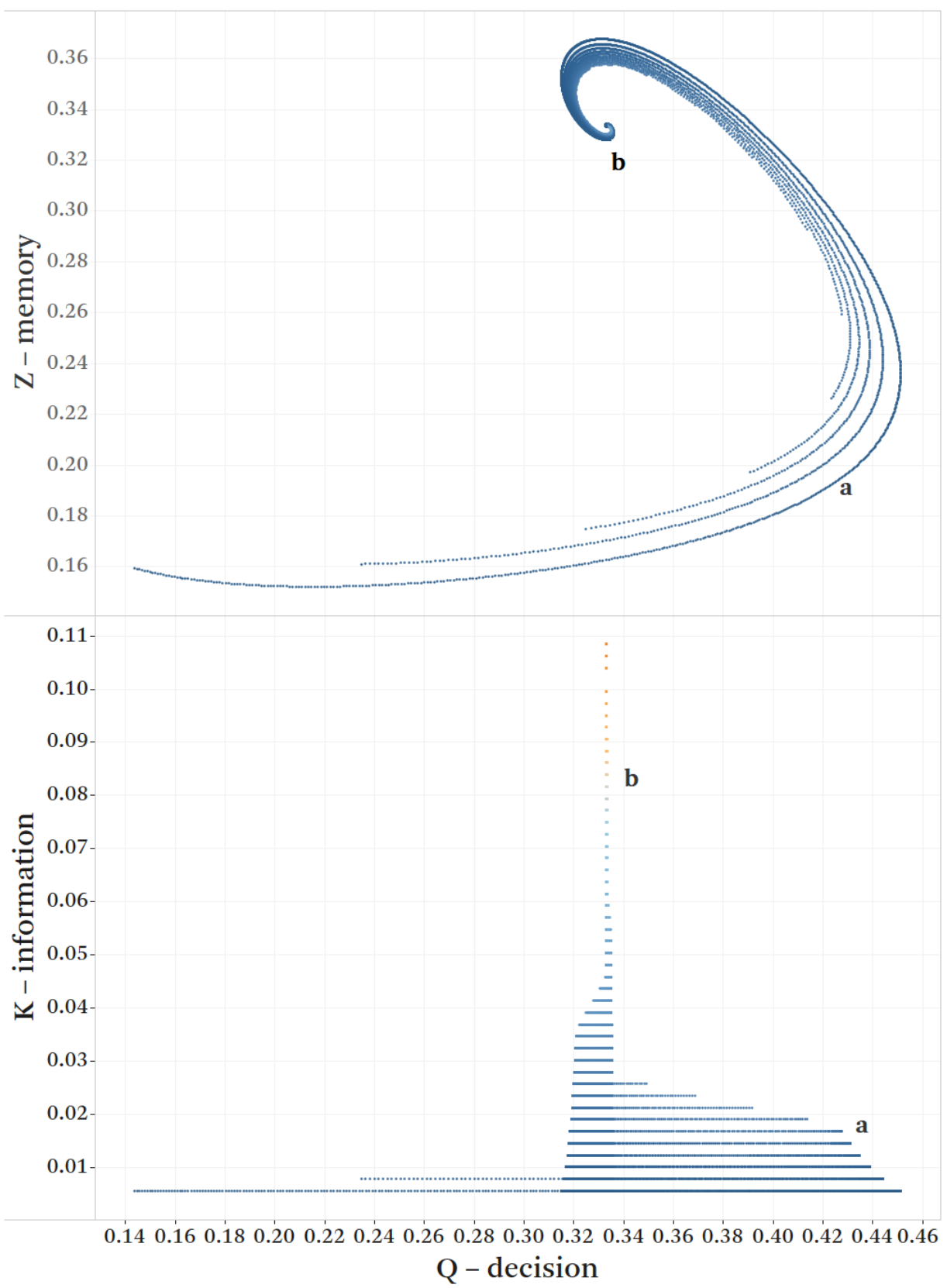

Fig. 4. Short Term Memory and Sensory Memory Channels

It is clear from Fig. 4 (a) that the number of distinctive channels in the short-term memory processing is between 5 and 7 after which the subsequent lines become blurred together and difficult to separate. This is supported by the experimental observation which estimates these at 7 plus minus 2 (Miller, 1956).

An illustration of information grouping or chunking is shown in Fig. 5 (a). During the short memory processing, the attractor forms distinctive groupings of points (Simon \& Chase, 1973). The number of points in each group increases with $\mathrm{k}$, thus a person associates additional portions of information during the process to facilitate the progress of information storing. 


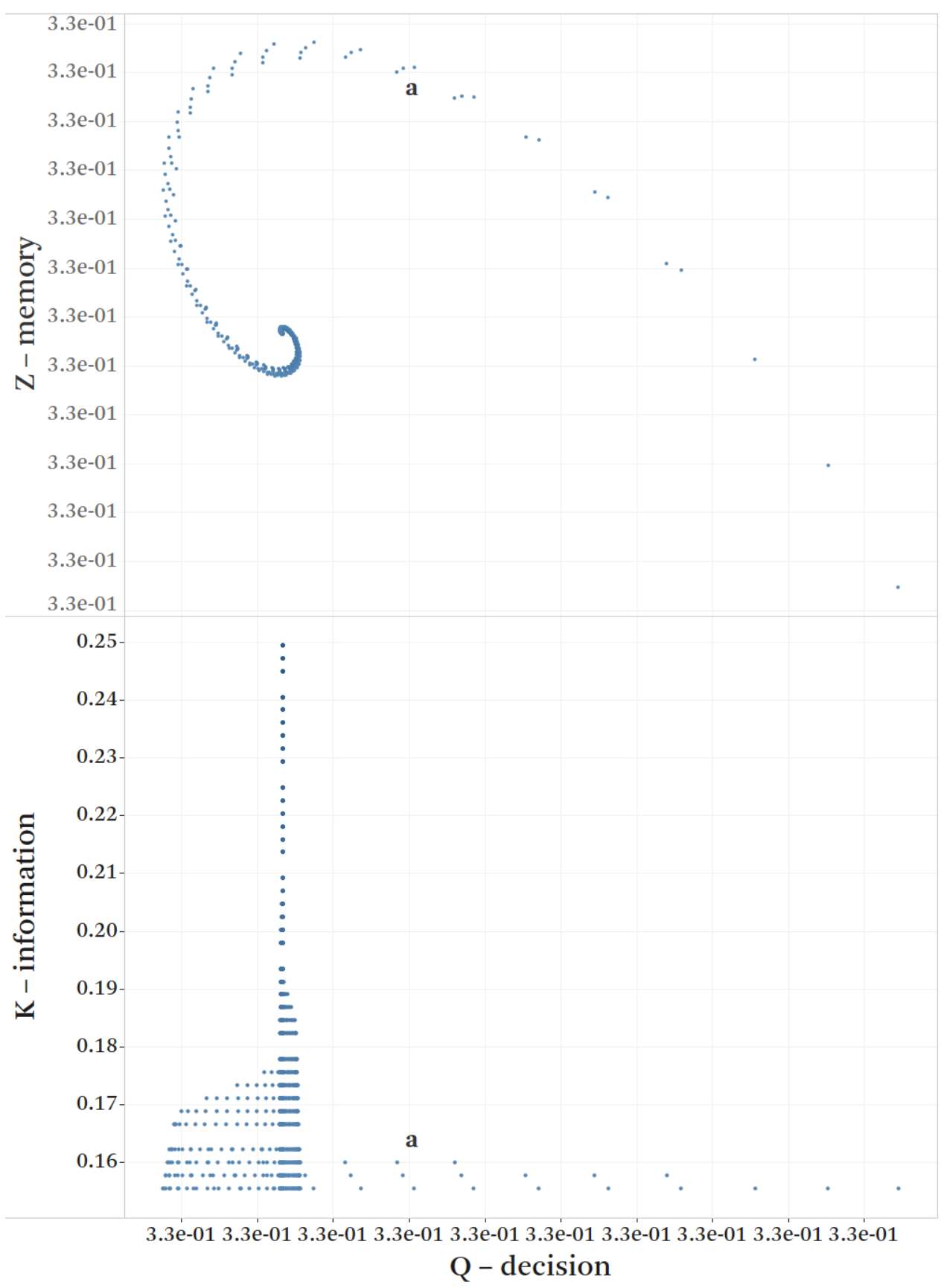

\section{Fig. 5. Chunking - Information grouping}

Fig. 6 illustrates the full 3-parameter attractor as defined by (4abc). The attractor is drawn for ten different initial conditions allowing for a fuller picture to be presented. (a) relates to the short-term memory, (b) to processing which includes maintenance rehearsal, self-referencing (Symons \& Johnson, 1997; Rogers, Kuiper \& Kirker, 1977), overlearning (Driskell, Willis \& Copper, 1992) and (c) to long-term memory storage (Nickerson \& Adams, 1979). The long-term memory storage is implemented through compartmentalization, which is manifested as separate curved orbits and through encoding which relates to the packing of information points along each orbit. Thus, compartmentalization is related to individual orbits while encoding is expressed through placing related fragments of information next to each other on the same orbit.

In cognitive science, compartmentalization represented here as orbits of the attractor is expressed through categories that create networks of associated memories. In this respect, categories may be positioned close to the bifurcated arm of the attractor, or be more extensively spanned and expressed through a long waving and elaborate curving line further away. Also, denoted as (c) in Fig. 6 categories may be abstract and removed far from the 
bifurcated line. In this regard, a notion of an abstract concept is associated with the distance and remoteness from the bifurcation. This agrees with the model behaviour with a relatively small number of initial points progressing to a full attractor manifestation. Thus, the ability to think in abstract terms utilizes the fullness of memory capabilities.

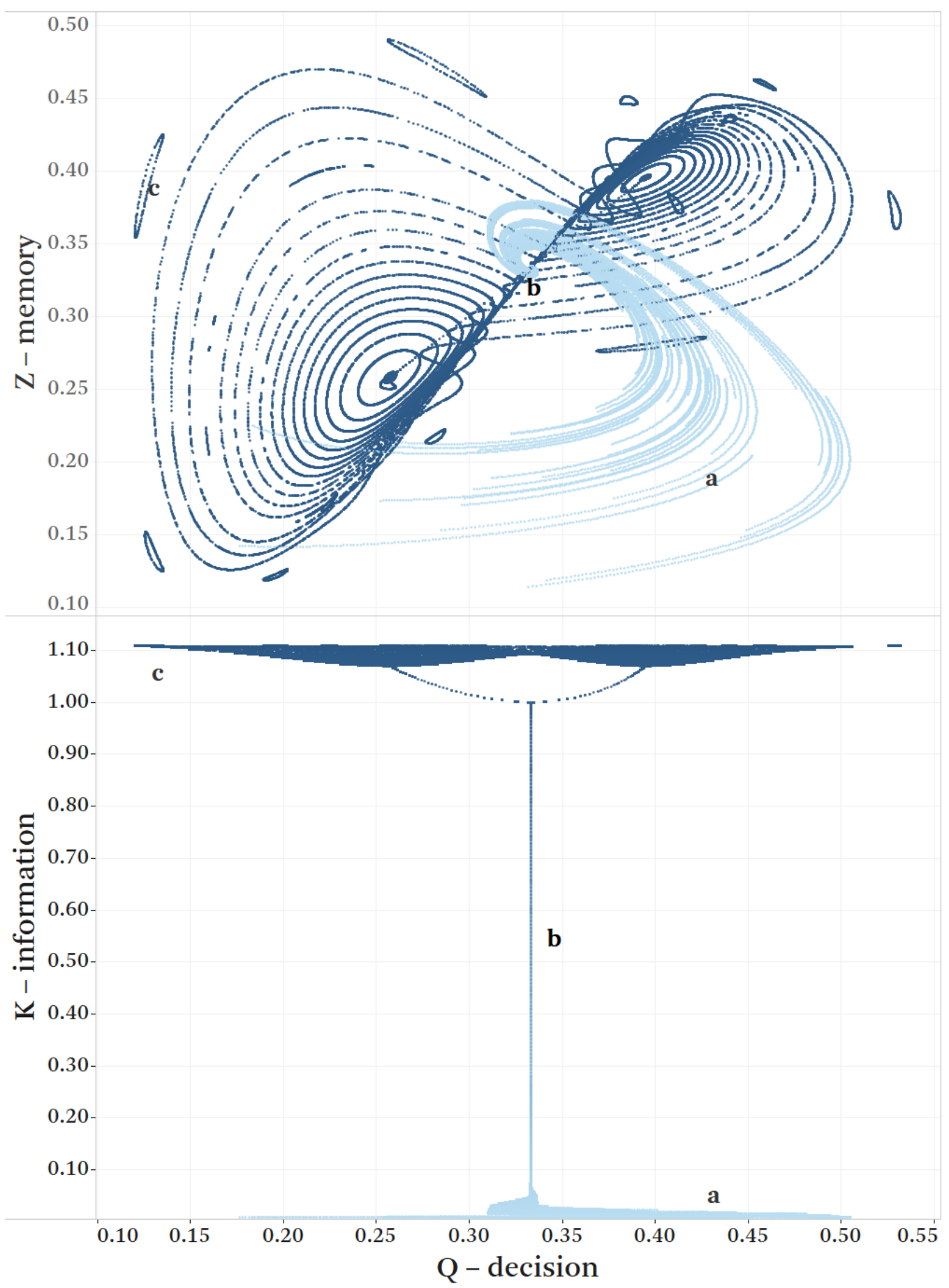

Fig. 6. A three-parameter strange attractor

\section{Four Parameter Model - understanding}

The fourth dimension of information is introduced through correlations. This concept is the most abstract of the four as it does not relate to external information gathering but instead, it relates to the way information is interconnected within the memory. Adding this fourth sequence to the model allows for the introduction of the 
concept of understanding. The memory points connected through (5d) may be spaced by a large distance. This is due to the general property of bifurcated attractors which connect arbitrarily separated points. Thus understanding connects facts belonging to different and sometimes unrelated categories.

Long-term memory stores information on the attractor's orbits joining related items together as categories. These are distinct and separated from each other. A phenomenon of cross-linking among the different categories is perceived as understanding. That is, understanding is an ability to connect information fragments that are located in different categories. In this form, the attractor expresses itself through collections of points, which cut across the memory orbits. The formulation of the attractor is provided through (5abcd).

$$
\begin{aligned}
& q_{n+1}=P(q \mid x)=\frac{P(q \cap \mathrm{x})}{P(x)}=\frac{-k q_{n} x_{n} \ln \left(q_{n} x_{n}\right)}{x_{n}}=-k q_{n} \ln \left(q_{n} x_{n}\right) \\
& p_{n+1}=P(p \mid q)=\frac{P(q \cap p)}{P(q)}=\frac{-k q_{n} p_{n} \ln \left(q_{n} p_{n}\right)}{q_{n}}=-k p_{n} \ln \left(q_{n} p_{n}\right) \\
& z_{n+1}=P(z \mid p)=\frac{P(\mathrm{z} \cap p)}{P(p)}=\frac{-k z_{n} p_{n} \ln \left(z_{n} p_{n}\right)}{p_{n}}=-k z_{n} \ln \left(z_{n} p_{n}\right) \\
& x_{n+1}=P(x \mid z)=\frac{P(\mathrm{x} \cap z)}{P(z)}=\frac{-k x_{n} z_{n} \ln \left(x_{n} z_{n}\right)}{z_{n}}=-k x_{n} \ln \left(x_{n} z_{n}\right)
\end{aligned}
$$

Similarly to (3a) and (3b), the normalization is achieved by dividing by the sum of all variables.

Fig. 7 illustrates the attractor at $\mathrm{k}$ values ranging between 1.05 and 1.10. The attractor is elaborate in all regions with extensive chaos above $\mathrm{k}=1.46$. It also displays complex behaviour at very low values of $\mathrm{k}$. In the region where long-term memory is stored, the attractor forms short line fragments that run across the memory lines.

In some respects, the attractor resembles the three-dimensional one. At very low values of $\mathrm{k}$, that is under 0.03 , the attractor expands and forms multidimensional warped surfaces when each pair of variables is plotted against the other. Therefore, in this region, the attractor forms a twisted multidimensional hyperspace. As the low k-region corresponds to the short-term memory, the attractor's behaviour corresponds to instantaneous perception, topic recognition and rapid subject placement observed among intelligent people.

At larger $\mathrm{k}$, the attractor forms trajectories that resemble two-dimensional lines in Fig. 2, more than the straight line formed by the three-parameter attractor in Fig. 6. Moreover, some trajectories terminate prematurely, which corresponds to the effect observed in the three-parameter model. Thus, like memory, understanding undergoes processing and may terminate prematurely.

There is a cutoff point at $\mathrm{k}=1.33$ where all surviving trajectories join either as a singular curve or become zero. Following this, the non-null line funnels at $\mathrm{k}=1.422$. Chaos sets in between $\mathrm{k}=1.464$ and 1.476.

The four-dimensional attractor extends beyond the range of the three-parameter one. The latter terminates at $\mathrm{k}=1.107$ while the former extends to $\mathrm{k}=1.476$. Thus, with the supply of information, understanding may expand beyond the limits of long-term memory, accounting for creativity and discovery. 


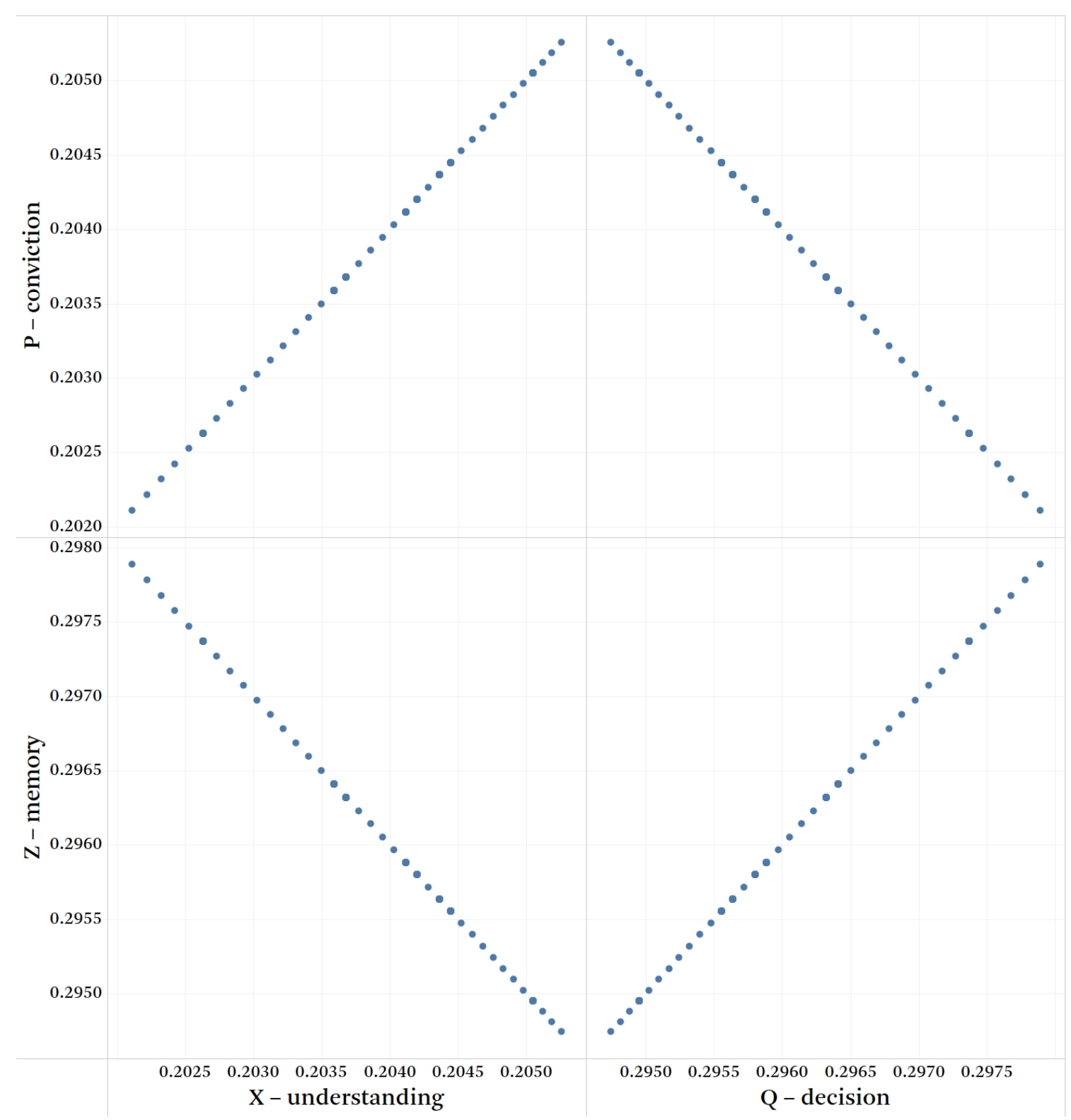

Fig. 7. Four-parameter attractor in the 1.05 to $1.10 \mathrm{k}$ range - understanding in the long-term memory region

\section{Conclusion}

The paper is opened with a discussion of a problem being faced by all researchers. The interpretation of observation and measurement. The ability to assign a truthful interpretation to any measurement challenges all researchers in all fields. In the case of cognitive research, a separation between the properties of information and the behaviour of a mind is particularly difficult due to the intrinsic complexity of both.

The model presented in the paper allows for the reproduction of numerous cognitive phenomena without referencing the workings of a human mind. The emphasis is on the properties of information, which is treated as an independently existing entity, and which is allowed to interact with itself within a multi-dimensional realm. It is astonishing that many of the effects described through the model, align with observations provided by cognitive research. On the other hand, there are numerous aspects of the workings of the mind which have not been touched by the model.

Thus, the one-parameter version of the model looks at the implicit memory but cannot consider explicit memory. The processing and storage of information are considered through the three-parameter model but the retrieval is not. Aspects of memory like the duration of storage and distortions due to the subsequent information fragments being stored are not addressed by the model. 
The most surprising is the ability of information to self-organize and the concept of understanding. These are the effects of imposing multidimensionality on the entropic formulae.

The field immediately impacted by this work is cognitive research. The way experiments are set and the results interpreted is influenced by these findings. The ability to separate the information related aspect of an experiment from the workings of a mind has an impact on those who study properties of information in its abstract form. Moreover, as this work is expressed through computer simulations, it enriches artificial intelligence studies providing new avenues for investigation.

Another group of scholars affected by this work are educators. Here, the interdependence of the four dimensions of information is presented. This in turn allows for the expansion of understanding of how learning is achieved. Every person displays different strengths in each of the information dimensions. Some are more inclined to factual memorization while others develop strengths in other dimensions. Based on the understanding provided here, educators can tweak their approaches to optimize the effectiveness of teaching.

Finally, while cognitive researchers and educators are directly affected by this research, commerce is also influenced. For example, advertisers are keen to see how and to what extend convictions influence memorization. This is important so to optimize advertising campaign success rates.

\section{References}

Atkinson, R. C., \& Shiffrin, R. M. (1968). Human memory: A proposed system and its control processes. Psychology of learning and motivation, 2(4), 89-195.

Caticha, A. (2011, March). Entropic time. In AIP Conference Proceedings (Vol. 1305, No. 1, pp. 200-207). American Institute of Physics. https://doi.org/10.1063/1.3573617

Caticha, A. (2015). Entropic dynamics. Entropy, 17(9), 6110-6128. https://doi.org/10.3390/e17096110

Chase, W. G., \& Simon, H. A. (1973). Skill in chess. American Scientist, 61(4), 394-403.

Cowan, N., Lichty, W., \& Grove, T. R. (1990). Properties of memory for unattended spoken syllables. Journal of Experimental Psychology: Learning, Memory, and Cognition, 16(2), 258. https://doi.org/10.1037/02787393.16.2.258

Driskell, J. E., Willis, R. P., \& Copper, C. (1992). Effect of overlearning on retention. Journal of Applied Psychology, 77(5), 615. https://doi.org/10.1037/0021-9010.77.5.615

Feigenbaum, M. J. (1978). Quantitative universality for a class of nonlinear transformations. Journal of statistical physics, 19(1), 25-52. https://link.springer.com/article/10.1007/BF01020332

Hartman, N., Olsen, C., Lüscher, S., Samani, M., Fallahi, S., Gardner, G. C., ... \& Folk, J. (2018). Direct entropy measurement in a mesoscopic quantum system. Nature Physics, 14(11), 1083-1086. https://doi.org/10.1038/s4156-018-0250-5

Luque, B., Lacasa, L., Ballesteros, F. J., \& Robledo, A. (2011). Feigenbaum graphs: a complex network perspective of chaos. PLoS One, 6(9), e22411. https://doi.org/10.1371/journal.pone.0022411

Martyushev, L. M. (2017). On interrelation of time and entropy. Entropy, 19(7), 345. https://doi.org/10.3390/e19070345

Martyushev, L. M., \& Shaiapin, E. V. (2016). Entropic measure of time, and gas expansion in vacuum. Entropy, 18(6), 233. https://doi.org/10.3390/e18060233

Miller, G. A. (1956). The magical number seven, plus or minus two: Some limits on our capacity for processing information. Psychological review, 63(2), 81. https://doi.org/10.1037/h0043158

Nickerson, R. S., \& Adams, M. J. (1979). Long-term memory for a common object. Cognitive psychology, 11(3), 287-307. https://doi.org/10.1016/0010-0285(79)90013-6

Parrondo, J. M., Van den Broeck, C., \& Kawai, R. (2009). Entropy production and the arrow of time. New Journal of Physics, 11(7), 073008. https://doi.org/10.1088/1367-2630/11/7/073008

Rogers, T. B., Kuiper, N. A., \& Kirker, W. S. (1999). Self-reference and the encoding of personal information. https://psycnet.apa.org/record/2000-07436-008

Shannon, C. E. (1959). Probability of error for optimal codes in a Gaussian channel. Bell System Technical Journal, 38(3), 611-656. https://doi.org/10.1002/j.1538-7305.1959.tb03905.x

Sperling, G. (1960). The information available in brief visual presentations. Psychological monographs: General and applied, 74(11), 1. https://doi.org/10.1037/h0093759

Symons, C. S., \& Johnson, B. T. (1997). The self-reference effect in memory: a meta-analysis. Psychological bulletin, 121(3), 371. https://psycnet.apa.org/buy/1997-03609-003

Verdu, S. (1998). Fifty years of Shannon theory. IEEE Transactions on information theory, 44(6), 2057-2078. https://doi.org/10.1109/18.720531

Vilenchik, L. Z., \& Vilenchik, M. (2019). The Emergence and Evolution of the Universe. Journal of High Energy Physics, Gravitation and Cosmology, 5(3), 884-898. https://doi.org/10.4236/jhepgc.2019.53044 
Wissner-Gross, A. D., \& Freer, C. E. (2013). Causal entropic forces. Physical review letters, 110(16), 168702. https://doi.org/10.1103/PhysRevLett.110.168702

\section{To cite this article}

\section{APA}

Szweizer, M., \& Schlagbaum, R. (2021, February 28). Entropy-based model for cognitive systems. https://doi.org/10.31219/osf.io/2tv9j

\section{MLA}

Szweizer, Moshe, and Rivka Schlagbaum. "Entropy-based Model for Cognitive Systems." OSF Preprints, 28 Feb. 2021. Web.

\section{Chicago}

Szweizer, Moshe, and Rivka Schlagbaum. 2021. "Entropy-based Model for Cognitive Systems." OSF Preprints. February 28. doi:10.31219/osf.io/2tv9j. 